\title{
Parameter optimization of LS-SVM base on PSO prediction of field intensity in mine tunnel
}

\author{
Xunhong $\mathrm{Li}^{1, \mathrm{a}}$, Jinli $\mathrm{Wu}^{2, \mathrm{~b}}$ \\ ${ }^{1}$ College of Communication and Information Engineering, Xi'an University of Science and \\ Technology, Xi'an, 7100554, China \\ ${ }^{2}$ College of Communication and Information Engineering, Xi'an University of Science and \\ Technology, Xi'an, 7100554, China \\ a email: lixhong105@xust.edu.cn, bemail:809300635@qq.com
}

Keywords: mine tunnel; field intensity; prediction; PSO-LSSVM

\begin{abstract}
The least squares support vector machines to solve small sample, nonlinear show some advantages, is very suitable for prediction of complex field intensity in mine tunnel , but the choice of kernel function and parameters for predicting the results have greater impact .PSO optimizing LSSVM parameters can improve prediction accuracy and generalization ability of the model. In this paper, in order to improve accuracy of prediction of field intensity in mine tunnel, PSO optimize the parameters of LS-SVM algorithm is adopted to the prediction of field intensity in mine tunnel and the prediction results are compared with the results of normal LS-SVM and BP neural network. The simulation results show that the PSO-LSSVM prediction of field intensity in mine tunnel is more efficient and accurate. For complex prediction of field intensity in mine tunnel theory has guiding significance.
\end{abstract}

\section{Introduction}

The prediction of field intensity in mine tunnel is very important to explore mine tunnel transmission characteristics and to establishment of safe and effective communication system, with the complex construction of mine tunnel, there are many factors affect the transmission of signal, such as mine tunnel with complex structures, equipments. The result of electromagnetic wave signal distortion, attenuation, and severe multipath effects. This is the biggest obstacle of mine tunnel wireless communication system.

In recent years, the study of electromagnetic waves for the mine tunnel tendency of traditional theoretical research method and machine learning. The traditional theory mainly geometrical optics and mode analysis, mode analysis is a use and purely theoretical method of Maxwell's equations for solving equations, complex mine tunnel propagation environment boundary conditions are difficult to determine, narrow the scope of application; Geometrical optics refers primarily ray tracing method though not bound by the shape of the mine, but only for the transmission of high frequency electromagnetic waves, more complex in theory and computation. In recent years, SuyKens.J.A.K proposed the least squares support vector machine based on the structural risk minimization, LS-SVM has simple, good performance and fast .And other learning algorithm, and the performance of LS-SVM depends on the parameters of the kernel function. How to determine the optimal parameters has become one of the questions about improving LS-SVM learning and generalization [2]. Traditionally, feature selection and regression parameter optimization problem is usually studied separately, PSO optimizing LSSVM model is a kind of synchronous optimization algorithm.

PSO (Particle Swarm Optimization) to optimize the least squares support vector machine, show the advantages of fast convergence rate and high robustness [3]. In this paper, established mine tunnel prediction model based on the least squares support vector machine. PSO optimization method of LS-SVM parameters applied to the prediction model of mine tunnel. 


\section{Based on LS-SVM mine roadway modeling analysis}

Given training vectors $x_{i} \in R^{m}$ and a vector $y_{i} \in R$ Optimization LS-SVM algorithm is :

$$
\begin{aligned}
& \min J=\frac{1}{2} w^{T} w+\frac{1}{2} r \sum_{i=1}^{N} e_{i}{ }^{2} \\
& \text { s.t. } y_{i}=w^{T} \varphi\left(x_{i}\right)+b+e_{i} ; i=1, \ldots, N
\end{aligned}
$$

In the type: $\varphi(\bullet): R^{m} \rightarrow R^{m}$ is the nonlinear mapping from input space to the high dimensional feature space functions; $\gamma$ for regularization coefficient.

According to the objective function and constraint condition, establishments the Lagrange function:

$$
L=\frac{1}{2} w^{T} w+\frac{1}{2} r \sum_{i=1}^{N} e_{i}^{2}-\sum_{i=1}^{N} a_{i}\left\{w^{T} \varphi\left(x_{i}\right)+b+e_{i}-y_{i}\right\}
$$

In the type: $a_{i}$ are the Lagrange multipliers.

According KKT condition get:

$$
\left\{\begin{array}{c}
\frac{\partial L}{\partial w}=0 \Rightarrow w=\sum_{i=1}^{N} \alpha_{i} \varphi\left(x_{i}\right) \\
\frac{\partial L}{\partial b}=0 \rightarrow \sum_{i=1}^{N} a_{i}=0 ; \\
\frac{\partial L}{\partial e_{i}}=0 \rightarrow a_{i}=\gamma e_{i}, i=1, \ldots, N \\
\frac{\partial L}{\partial a_{i}}=0 \rightarrow w^{T} \varphi\left(x_{i}\right)+b+e_{i}-y_{i}=0, i=1, \ldots N
\end{array}\right.
$$

After the elimination of $w$ and $e$ to give the matrix equation:

$$
\left[\begin{array}{cc}
0 & 1_{V}^{T} \\
1_{V} & \Omega+\frac{1}{\gamma} I
\end{array}\right]\left[\begin{array}{l}
b \\
a
\end{array}\right]=\left[\begin{array}{l}
0 \\
y
\end{array}\right]
$$

In the type, $\Omega_{i j}=\varphi\left(x_{i}\right)^{T} \varphi\left(x_{j}\right)=K\left(x_{i}, x_{j}\right), i, j=1, \ldots, N \quad y=\left[y_{1}, \ldots, y_{N}\right]{ }^{T} ; 1_{v}=[1, \ldots, 1] ; \alpha=\left[a_{1}, \ldots, \alpha_{N}\right]^{T}$; I is a unit matrix ,the regression function is:

$$
y(x)=\sum_{i=1}^{N} a_{i} K\left(x, x_{i}\right)+b
$$

In the type, $k\left(x_{i}, x_{j}\right)$ is the kernel function, commonly used kernel function is linear kernel function and polynomial kernel function, the radial basis kernel function and so on. Usually in the absence of prior knowledge, as well as when the model complex nonlinear system, choose RBF better than other nuclear choice, Taking into account the electromagnetic wave propagation mine tunnel is complex nonlinear system, so chose the RBF function as the kernel function.

$$
\psi\left(x, x_{i}\right)=\exp \left\{-\left\|x-x_{i}\right\|^{2} / 2 \sigma^{2}\right\}
$$

RBF kernel function of the structure is simple, strong generalization ability parameters to be optimized only kernel parameter $\delta$ and regularization parameters $\gamma$, based on the research of the mine electromagnetic theory, determine the main factors influencing the mine roadway electromagnetic wave propagation including the carrier frequency, sectional area of the roadway, electrical parameters of coal and rock roadway wall roughness and other factors as LSSVM input factors. field strength decline is a output Establish the LSSVM model of field intensity in mine tunnel, using PSO optimization algorithm to select LS-SVM parameters $\delta$ and $\gamma$, 


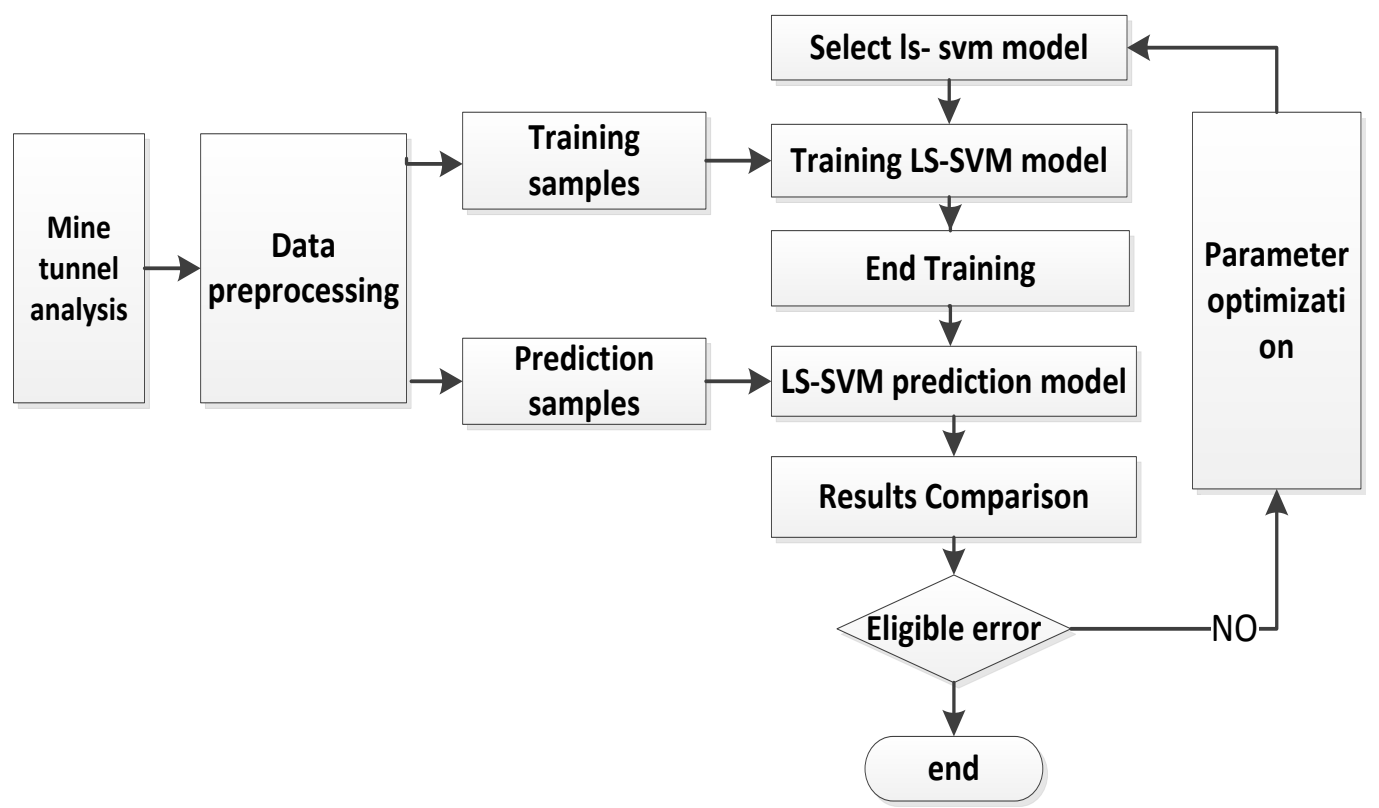

Fig1 Prediction of field intensity in mine tunnel base on LS SVM

\section{PSO optimize LSSVM algorithm}

Particle swarm algorithm is initialized to a set of particles (random solutions), and then through the iterative search for the optimal solution. Particles through the two extreme update yourself: one is the particle so far to find the optimal value, called individual extreme value; The other one is the whole particle swarm so far to find the optimal value, called the global extreme [4] particle update formula is:

$$
\begin{gathered}
V^{t+1}=w V^{t}+c_{1} \times r_{1} \times\left(p^{t} \text { best }-x^{t}\right)+c_{2} \times r_{2} \times\left(g^{t} \text { best }-x^{t}\right) \\
X^{t+1}=X^{t}+V^{t+1}
\end{gathered}
$$

In the type, $t$ is the iteration number; $v_{t}$ is particle flight speed of the $t$ generation. $\mathrm{w}$ is the inertia weigh.

RBF parameters are very important to performance prediction model in mine tunnel .it's extremely important to determine the parameters of the $\delta$ and $\gamma$,PSO has fast convergence and global convergence ability, we can optimize the penalty factor and kernel function parameters, To some extent, to avoid the blindness of artificial selection parameter.PSO optimize the LS-SVM parameters greatly improving the LS-SVM generalization.

PSO algorithm to optimize LSSVM parameters, the following steps:

(1) Initialize the PSO parameters: $\mathrm{c} 1=\mathrm{c} 2=1.4959$, the maximum number of iterations $\mathrm{Gmax}=$ 300 , the number of population particle swarm $\mathrm{N}=30$, the inertia weight $\mathrm{w}=0.9$.

(2) With each particle corresponding LS-SVM learning samples forecast, respectively; to give a prediction error of each particle current position, and as the fitness value .Compare current fitness value of the particle itself and the optimal fitness value, which is better, then the particles as the current optimal position.

(3) To compare the fitness value of the particle value and he fitness value of groups, if better, the particle position as the group position. And using the formula (8) updating the particle velocity and position.

(4) Check whether meet the end condition (to preset maximum number of iterations or prediction accuracy), if meet the end conditions, find out the optimal solution; otherwise the jump step 2), continue to a new round of the search. 
The PSO optimized parameters $\delta$ and $\gamma$ applied to the prediction model of field intensity in mine tunnel base on LS-SVM .compare the output and prediction output to determine the merits of the model. If the error is within the allowable range if the error is too large, adjust PSO optimization LS-SVM algorithm to adjust the accuracy of the model, up to meet the requirements until.

\section{The results of simulation analysis}

Data for a mine tunnel by actual measurement, the data in reference [5]. The actual measured field strength fading distribution as follows:



Fig 2.The field intensity distribution

In this paper, establishing the intensity prediction model in mine tunnel base on LS-SVM., the PSO is used to optimize the parameters of the LS-SVM At the same time, this paper uses the normal LS-SVM optimization method and the BP neural network prediction model to forecast the field intensity in mine tunnel Using mat lab software to simulate, The simulation results are as follows:

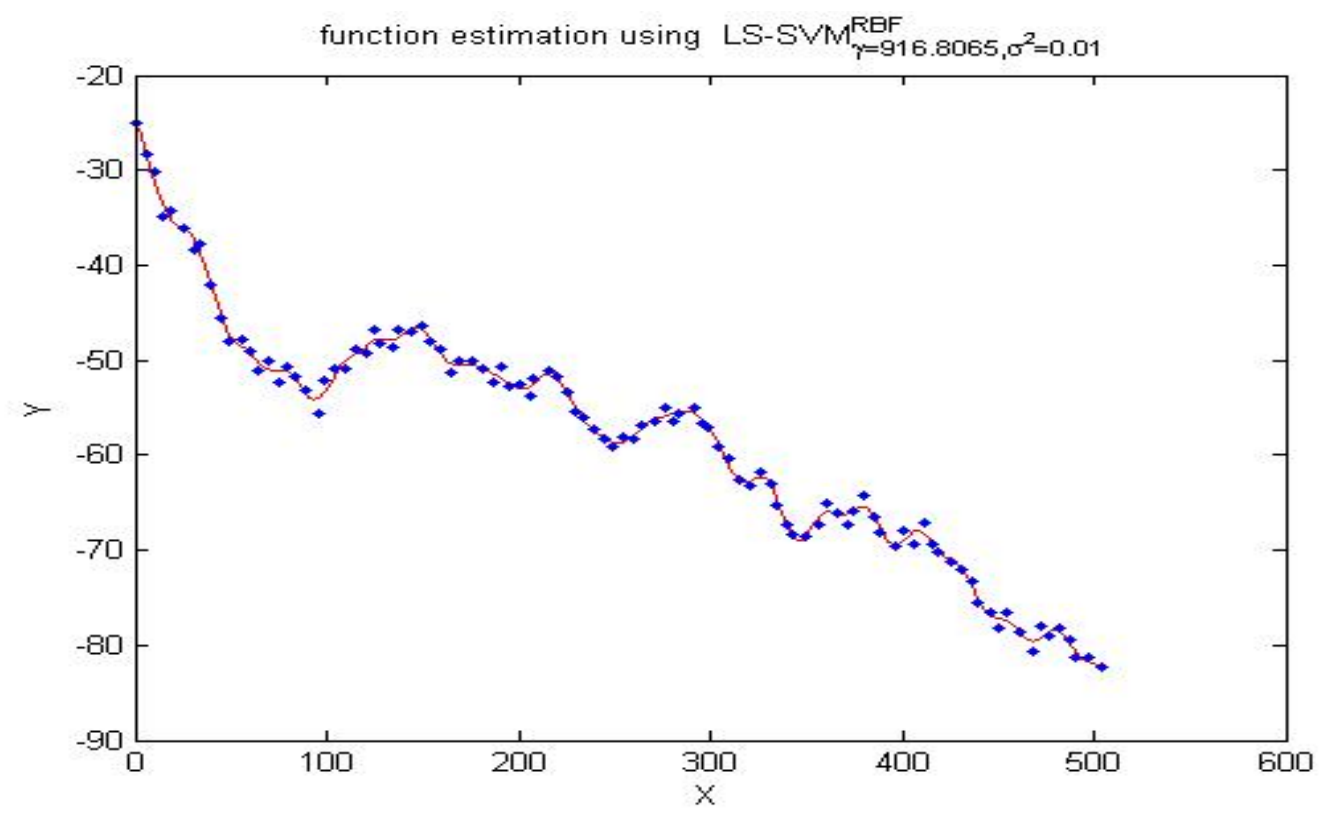

Fig 3. PSO optimization LS-SVM model 


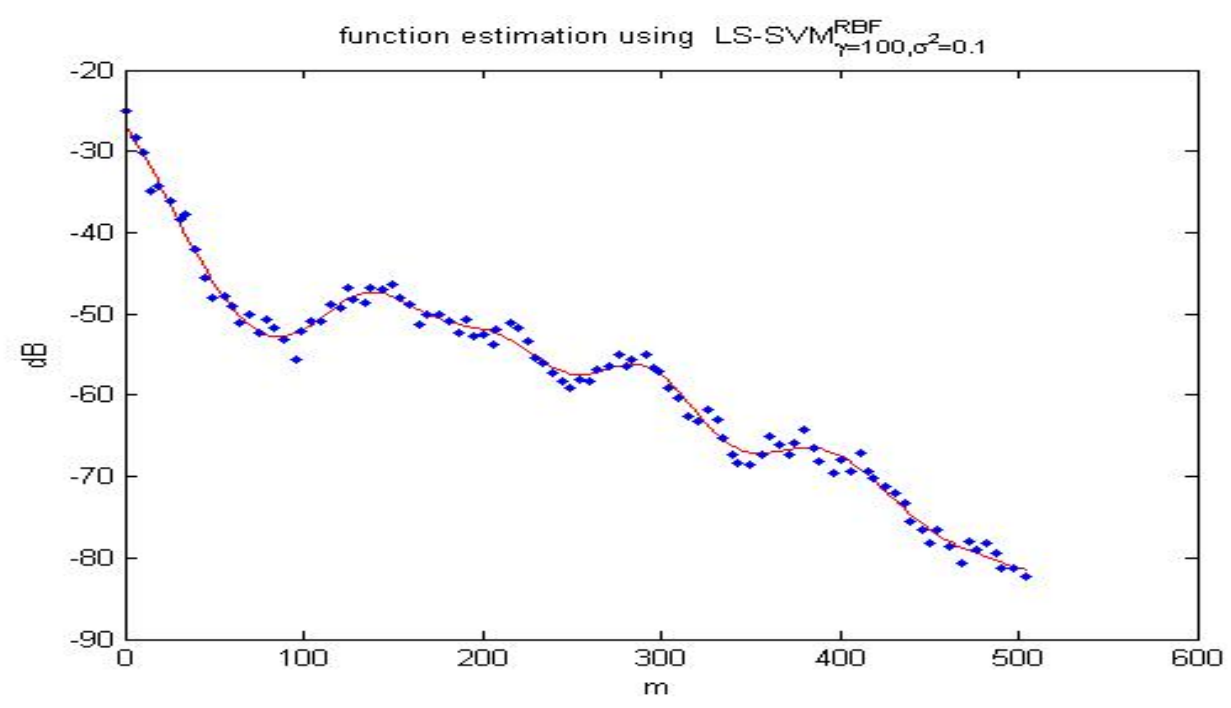

Fig 4.normal optimization LS-SVM model

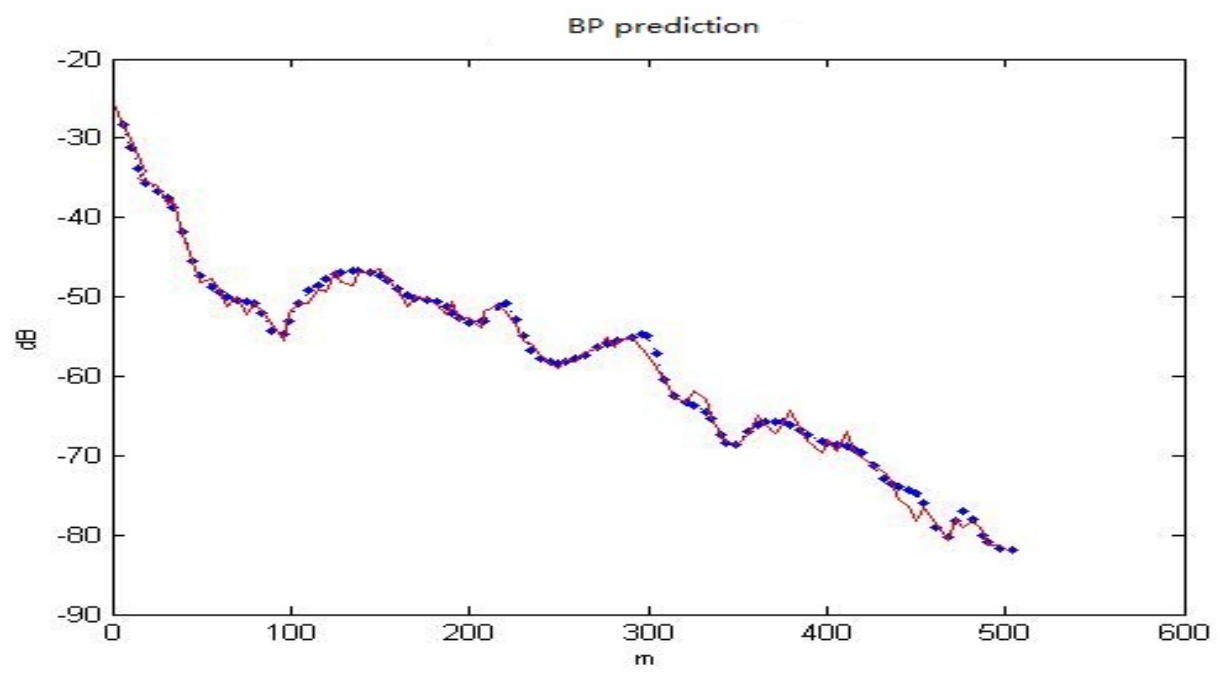

Fig 5.BP prediction model


BP prediction error

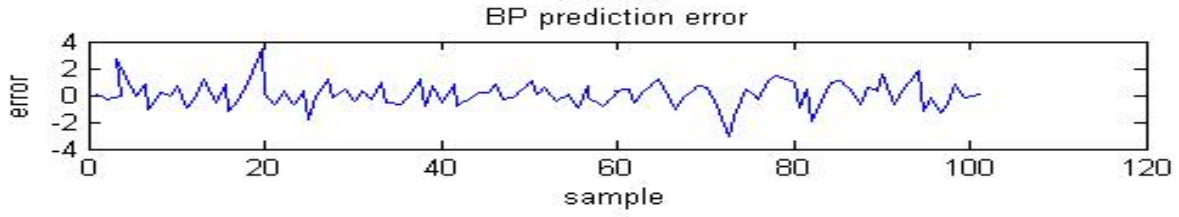

Fig 6 Comparison error 
Simulation results show that: the prediction in the measured data of the mine tunnel field intensity .PSO optimization LS-SVM result in a more accurate approximation of the true value, although BP neural network implicit node 20 can be a good approximation of the mine tunnel intensity true value, but in many points it is over-fitting. At the same time, the prediction error of three algorithms showed that: the PSO is used to optimize the parameters of the LS-SVM has showed certain advantages in terms of accuracy With the traditional LS - SVM model compared with the BP neural network possesses the advantages of higher prediction precision.

\section{Conclusion}

Based on the least squares support vector machine (SVM) is established to forecast in mine tunnel intensity, PSO algorithm to optimize the LS -SVM parameter method is applied to prediction model of the mine tunnel intensity, and with the traditional LS - SVM prediction model and BP neural network prediction model is compared, the result more accurate, the experiment showed that PSO to optimize the LS - SVM model parameters for complex mine tunnel field intensity forecast more practical.

\section{Acknowledgement}

In this paper, the research was sponsored by the Natural Science Basic Research Plan in Shaanxi Province of China(Program No.S2015YFJM1734)

\section{References}

[1]Yan G F ,Zhang X P, Wang S Y 2014.Remote-sensing retrieval of suspended solids base on improved PSO-LSSVM at Harbin section of the Songhua River[J].Acta Scientiae Circumstantiae,34(8):2148-2156

[2]YAO Quan-zhu, CAIJie. Feature selection and LS-SVM parameters optimization algorithm based on PSO[J]. Computer Engineering and Applications, 2010, 46(1):134-136.

[3]JIA Rong,ZHANG Yun,HONG Gang, Parameter optimization of least squares support vector machine base on improved particle swarm optimization in fault diagnosis of transformer[J].Power System Protection and Control.,2010, 17:121-124+152.

[4]LIU Guodong, ZHANG Zhifeng. An Improved Particle Swarm Optimization Algorithm and Genetic. Algorithm. Fire Control Command Control. 2010, v.35S1:150-151+154.

[5]WANG Anyi, GUO Shikun. Prediction of field intensity in mine tunnel base on LS-SVM[J]Industry and Mine Automation, 2014,10:36-40 